\title{
COMPARAÇÃO ENTRE SOLUÇÃO SALINA 0,9\% E CRISTALOIDES BALANCEADOS: REVISÃO BIBLIOGRÁFICA
}

\author{
COMPARISON BETWEEN SALINE AND BUFFERED SOLUTIONS: \\ BIBLIOGRAPHIC REVIEW
}

Diego Alex de Araújo Costa MD; Wallace Andrino da Silva, MD, PhD, TSA ${ }^{2}$

1. Médico anestesiologista formado pelo programa de anestesiologia do Hospital Universitário Onofre Lopes.

2. Médico anestesiologista do Hospital Universitário Onofre Lopes, residência médica em anestesiologia e doutorado pela Faculdade de Medicina da Universidade de São Paulo, Título Superior em Anestesiologia (TSA) pela Sociedade Brasileira de Anestesiologia (SBA).

Artigo produzido na Divisão de Anestesiologia do Hospital Universitário Onofre Lopes (UFRN).

Suporte financeiro: Não.

Conflito de interesse: Não.

Endereço: Hospital Universitário Onofre Lopes, Divisão de Anestesiologia. Av. Nilo Peçanha, 620, $3^{\circ}$ subsolo, Petrópolis, Natal/RN CEP:59012-300.

Submetido: maio 07; Aceito após revisão: maio 12, 2020.

\section{ABSTRACT}

An important milestone in medicine was the development of intravenous fluids. It has an immediate impact on the outcomes of diseases, such as cholera and polytrauma patients. Its importance triggered interest in the study of the physiology and anatomy of macro and microcirculation, the creation of new fluids, as well as the impact of the type of solutions on clinical outcome. Saline solution, more known as physiological saline or normal saline solution, is one of the oldest and most used solutions in the world. Buffered solutions, such as Plasma-lyte ${ }^{\circledR}$, Lactate Ringer, among others, which uses weak buffers and anions in its composition, proved to be more physiological, with better clinical outcomes, in a wide variety of patients. The discoveries of the anatomy and physiology of microcirculation, hydroelectrolytic and acid-base alterations specific to each disease, hyperchloremia and its harmful effects brought a wide and important discussion in the indication of each crystalloid. The type and amount of fluid to be administered in the perioperative period have a direct impact on the outcome. Buffered solutions have been presented as a superior alternative to $0.9 \%$ saline in the perioperative context. 
Comparison between saline and buffered solutions: bibliographic review

Costa DAA; Silva WA

Key words: Crystalloids. Saline solution. Lactate ringer. Plasma-lyte ${ }^{\circledR}$. Buffered solutions. Perioperative.

\section{RESUMO:}

Um marco importante na medicina foi o desenvolvimento dos fluidos intravenosos. Obteve-se impacto imediato nos desfechos de doenças, como a cólera, e nos pacientes politraumatizados. Sua importância desencadeou um interesse pelo estudo da fisiologia e anatomia da macro e microcirculação, criação de novos fluidos, bem como o impacto do tipo das soluções sobre os desfechos clínicos. A solução salina, popularizada como solução fisiológica ou normal, é uma das soluções mais antigas e mais utilizadas no mundo. As soluções balanceadas, como o Plasma-lyte ${ }^{\circledR}$, Ringer Lactato, entre outros, que utilizam tampões e ânions fracos na composição, começaram a ser apresentadas e se mostraram mais fisiológicas e com desfechos clínicos melhores em uma variedade extensa de pacientes. As descobertas da anatomia e fisiologia da microcirculação, alterações hidroeletrolíticas e ácido-básicas específicas de cada doença, hipercloremia e seus malefícios trouxeram uma discussão ampla e importante na indicação de cada cristaloide. $\mathrm{O}$ tipo e a quantidade de fluido a ser administrada no perioperatório têm impacto direto no desfecho dos pacientes. As soluções balanceadas vêm se apresentando como alternativa superior à solução salina $0,9 \%$ no contexto perioperatório.

Palavras-chave: Sepse. Pneumoperitôneo. Citocinas. Procalcitonina. Proteína C reativa. Inflamação.

\section{INTRODUÇÃO}

A chegada da pandemia de cólera em Sunderland, cidade localizada no nordeste da Inglaterra, em outubro de 1831, estimulou o desenvolvimento das primeiras terapias com fluidos intravenosos ${ }^{1}$. A composição da solução salina a $0,9 \%$ foi mencionada primeiramente por Jakob Hamburger em meados de 1890. É desconhecido a origem do termo "normal" ou "fisiológico" quando fazemos referência a solução salina $0,9 \%$, já que a solução não é fisiologicamente "normal".

A solução salina $0,9 \%$ possui altíssimas concentrações de cloro e, ainda, possui uma diferença de íons fortes (DIF) diferente do plasma. De acordo com a abordagem de Stewart dos distúrbios ácido-básicos, o pH de um fluido é parcialmente determinado pela DIF, que consiste na soma das concentrações dos cátions fortes (por exemplo: potássio, sódio e magnésio) subtraindo-se a soma das concentrações dos ânions fortes (por exemplo: cloro e lactato). A DIF do líquido extracelular (LEC) é aproximadamente $40 \mathrm{mEq} / \mathrm{L}$, enquanto a DIF da solução salina a 0,9\% é 0 . A infusão de solução salina $0,9 \%$ reduz a DIF do LEC, provocando acidose metabólica hiperclorêmica ${ }^{2}$. Sidney Ringer 
Comparison between saline and buffered solutions: bibliographic review Costa DAA; Silva WA

realizou estudos in vitro sobre a influência da composição dos cristaloides na contratilidade cardíaca, os quais demonstraram potenciais benefícios na adição de outros constituintes inorgânicos nas soluções salinas. Alexis Hartmann modificou a solução de Ringer acrescentando o lactato de sódio com o objetivo de balancear a concentração de cloro e tornar a solução mais próxima do fisiológico ${ }^{2}$. Diferentemente das soluções salinas $0,9 \%$, as soluções balanceadas possuem lactato, acetato, malato e/ou gluconato, os quais agem como precursores de bicarbonato. A solução de ringer lactato possui DIF de 29, se aproximando mais da DIF do plasma que a solução salina $0,9 \%$. Em contraste, o Plasma-lyte ${ }^{\circledR}$ possui uma DIF de $50 \mathrm{mEq} / \mathrm{L}$. Apesar do fato das soluções balanceadas tentarem mimetizar a composição do plasma, nenhuma é perfeitamente fisiológica (ver tabela 1$)^{2}$.

Tabela 1 - Características de soluções cristaloides comuns comparadas ao plasma humano

\begin{tabular}{|c|c|c|c|c|c|}
\hline & $\begin{array}{l}\text { Plasma } \\
\text { Humano }\end{array}$ & $\begin{array}{l}\text { Salina } \\
0,9 \%\end{array}$ & $\begin{array}{l}\text { Ringer } \\
\text { Simples }\end{array}$ & $\begin{array}{l}\text { Ringer } \\
\text { Lactato }\end{array}$ & $\begin{array}{c}\text { Plasma } \\
\text { Lyte }^{\circledR}\end{array}$ \\
\hline Sódio (mmol/L) & $136-145$ & 154 & $147-147,5$ & 130 & 140 \\
\hline Potássio (mmol/L) & $3,5-5,0$ & & $4,0-4,47$ & 4 & 5 \\
\hline Magnésio (mmol/L) & $0,8-1,0$ & & & & 1,5 \\
\hline Cálcio (mmol/L) & $2,2-2,6$ & & $4,47-4,5$ & 3 & \\
\hline Cloro (mmol/L) & $98-106$ & 154 & $155,5-156$ & 109 & 98 \\
\hline Acetato (mmol/L) & & & & & 27 \\
\hline Gluconato (mmol/L) & & & & & 23 \\
\hline Lactato (mmol/L) & & & & 28 & \\
\hline DIFe (mEq/L) & 42 & 0 & 0 & 28 & 50 \\
\hline $\begin{array}{c}\text { Osmolaridade Teórica } \\
\text { (mosmol/L) }\end{array}$ & 291 & 308 & $309-310$ & 273 & 295 \\
\hline $\begin{array}{l}\text { Osmolalidade atual ou } \\
\text { mensurada (mosmol/Kg } \\
\text { Água) }\end{array}$ & 287 & 286 & & 256 & 271 \\
\hline $\mathrm{pH}$ & $7,35-7,45$ & $4,5-7,0$ & $5,0-7,5$ & $5,0-7,0$ & $4,0-8,0$ \\
\hline
\end{tabular}

O uso da solução salina $0,9 \%$ vem se tornando cada vez mais restrito devido ao risco aumentado de causar hipercloremia. Hipercloremia tem sido estudada em crianças com choque séptico meningocócico, como causadora de acidose metabólica pósressuscitação volêmica ${ }^{3}$. Esta observação foi reforçada 2 anos depois em um estudo com pacientes adultos com choque séptico ${ }^{4}$. Pedoto e colaboradores observou importante papel da acidose hiperclorêmica na indução do aumento da atividade da enzima oxido nítrico sintetase e, consequentemente, hipotensão sistêmica e vasoplegia ${ }^{5-7}$. Ainda, a redução do $\mathrm{pH}$ devido ao excesso de cloro parece estar relacionada a efeito próinflamatório, redução da função renal, lesão pulmonar, redução da motilidade intestinal e aumento da mortalidade na sepse ${ }^{8}$. 
Comparison between saline and buffered solutions: bibliographic review Costa DAA; Silva WA

O conhecimento da interação dos fluidos entre os diferentes compartimentos corporais é fundamental para compreender as diferentes fisiopatologias às quais ela é submetida e como as diferentes osmolaridades e composições das soluções cristaloides, com suas propriedades físico-químicas e biológicas, podem colaborar com melhores desfechos clínicos. Essa revisão tem como objetivo analisar a literatura mais atual que relacione a escolha da solução mais apropriada para cada contexto clínico.

\section{COMPARTIMENTO DE FLUIDOS}

A água compõe cerca de $60 \%$ do peso corporal total no adulto médio, variando com a idade, gênero e composição corporal. A água corporal total (ACT) é dividida entre compartimentos de fluidos anatômicos e funcionais dentro do corpo, com uma grande divisão entre o líquido intracelular (LIC) e o líquido extracelular. 0 tamanho destes compartimentos e a sua composição muito divergente são mostrados na figura $1 \mathrm{e}$ tabela 2, respectivamente. O volume total do sangue compreende elementos extracelulares - compartimento plasmático e do subglicocálice - e intracelulares - células do sangue. Com a exclusão do compartimento não funcional do LEC - ossos e tecido conjuntivo -, a relação entre LIC e LEC funcional é de aproximadamente 2:1.

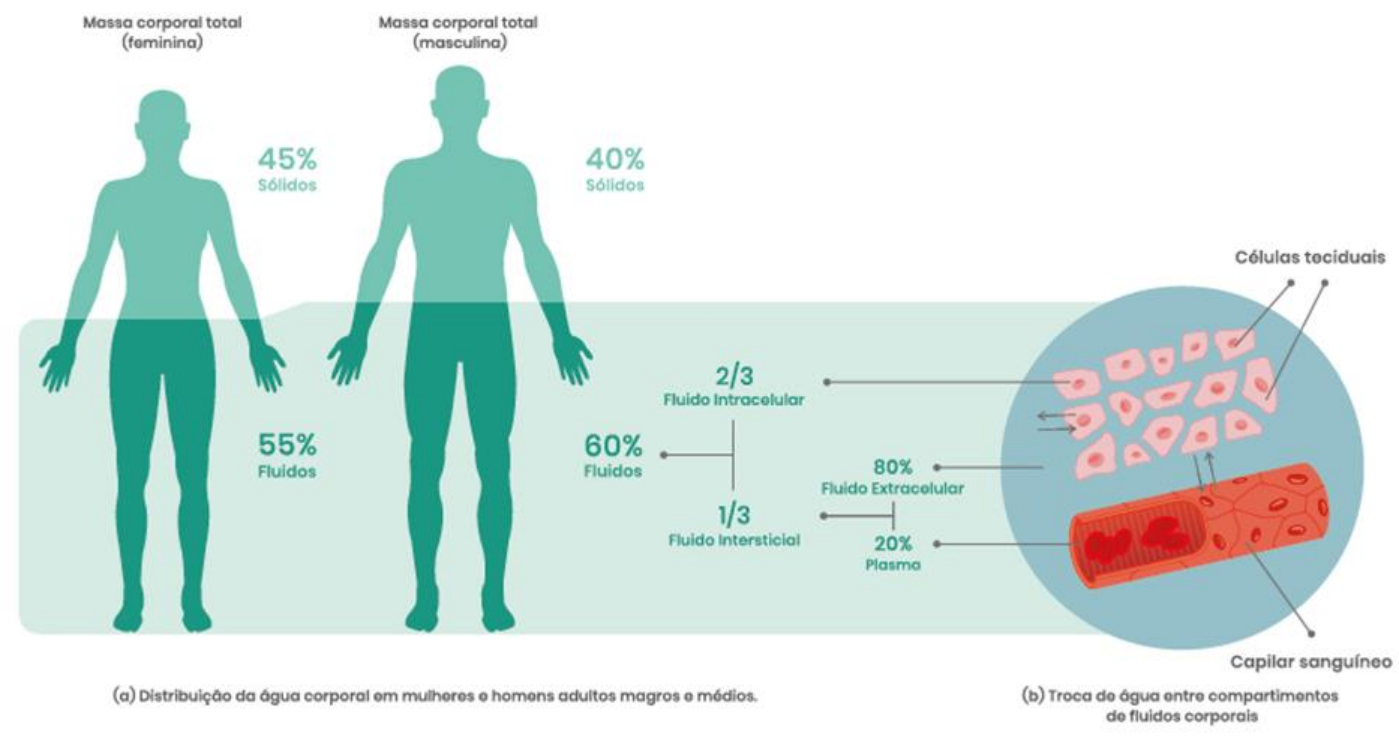

Figura 1 - Distribuição de água corporal e troca de água entre compartimentos 
Comparison between saline and buffered solutions: bibliographic review Costa DAA; Silva WA

Tabela 2 - Composição dos Compartimentos de Líquidos Intracelular e Extracelular (em $\mathrm{mOsm} / \mathrm{L}$ de água)

\begin{tabular}{|l|c|c|c|}
\hline & \multicolumn{2}{c}{ Extracelular } \\
\hline Cátions & Intracelular & Intravascular & Intersticial \\
\hline Sódio & \multicolumn{3}{c}{} \\
\hline Potássio & 10 & 142 & 145 \\
\hline Cálcio & 157 & 4 & 4 \\
\hline Magnésio & $0,5^{*}$ & 2,5 & 2,5 \\
\hline
\end{tabular}

\begin{tabular}{l|c|c|c|}
\hline Ânions & & & \\
\hline \multicolumn{1}{c|}{ Cloro } & 10 & 103 & 117 \\
\hline Bicarbonato & 7 & 25 & 27 \\
\hline Fosfato & 11 & 2 & 2 \\
\hline Sulfato & 1 & 0,5 & 0,5 \\
\hline Ácidos Orgânicos & 4 & 6 & 6 \\
\hline Proteínas & 1,2 & 0,2 \\
\hline $\begin{array}{l}* \\
\text { A concentração de Cálcio intracelular pode estar tão elevada quanto ao extracelular, mas seu } \\
\text { tamponamento mantém níveis reduzidos no intracelular. }\end{array}$ \\
\hline
\end{tabular}

Importante também é o entendimento das leis físico-químicas que regem o movimento de fluidos e eletrólitos, pois elas são responsáveis pelos diferentes mecanismos de manutenção das composições de cada compartimento.

\section{MOVIMENTO DE FLUIDO E ELETRÓLITOS}

A difusão é o processo pelo qual as partículas de soluto preenchem o volume do solvente disponível por movimento, a partir de áreas de alta para baixa concentração. A osmose é a passagem de solvente através de uma membrana semipermeável, com passagem deste das áreas de menor concentração para de maior concentração. A pressão hidrostática necessária para resistir ao movimento das moléculas é a pressão osmótica. Esta é uma das propriedades coligativas fundamentais de uma solução, ou seja, depende do número de partículas e não do tipo de partículas osmoticamente ativas numa solução, que podem ser moléculas completas ou íons dissociados. Os fluidos corporais não são soluções ideais, porque interações interiônicas reduzem o número de partículas livres para exercer o efeito osmótico. A pressão osmótica do plasma é de aproximadamente $5.545 \mathrm{mmHg}$.

Molalidade é o número de mols ( 1 mol = 6 × 1023 partículas de uma substância específica) presente em $1 \mathrm{~kg}$ de solvente. A osmolalidade pode ser usada para descrever soluções que contêm muitos tipos de partículas diferentes e é o número de osmoles (cada um contendo 6 × 1023 de qualquer tipo de partícula presente) presente em $1 \mathrm{~kg}$ de solvente. A osmolalidade corporal normal é de $285-290 \mathrm{mOsm} / \mathrm{kg}$ e é a mesma nos compartimentos intra e extracelulares, devido à livre circulação de água entre os 
Comparison between saline and buffered solutions: bibliographic review

Costa DAA; Silva WA

compartimentos que, consequentemente, impede o desenvolvimento de quaisquer gradientes osmóticos. A osmolaridade sérica é estimada por 2 vezes a concentração de sódio em $\mathrm{mEq} / \mathrm{L}$ (aproximação feita pela lei da eletroneutralidade) somada a concentração de glicose em $\mathrm{mg} / \mathrm{dL}$ dividida por 18 e concentração de uréia em $\mathrm{mg} / \mathrm{dL}$ dividida por 6 .

Tonicidade é a osmolalidade eficaz de uma solução em relação a uma membrana semipermeável em particular e leva em conta solutos que não exercem um efeito osmótico in vivo. Por exemplo, sódio e cloro não atravessam livremente as membranas celulares e, por conseguinte, exercem uma força osmótica eficaz entre as membranas, enquanto a uréia se difunde livremente através das membranas celulares e, portanto, não exerce um efeito osmótico. A glicose é considerada um osmol ineficaz, já que entra nas células por difusão facilitada estimulada pela insulina. A tonicidade é importante in vivo e é percebida pelos osmorreptores do hipotálamo. Podemos estimá-la subtraindo ureia e glicose da fórmula de osmolalidade.

A pressão oncótica é o componente de pressão osmótica total é devido aos colóides - isto é, as partículas de peso molecular elevado, predominantemente proteínas como albumina, globulinas e fibrinogênio. Da pressão total osmótica do plasma de $5.545 \mathrm{mmHg}, 25$ a $28 \mathrm{mmHg}$ são devidos à pressão oncótica no plasma. A carga negativa das proteínas tem a capacidade de reter uma pequena quantidade de sódio no plasma (efeito de Gibbs-Donnan), o que aumenta efetivamente a pressão oncótica acima do que seria previsível com base puramente por meio de cálculos de concentrações proteica. A albumina é a proteína mais abundante do plasma e é responsável por $65-75 \%$ da pressão oncótica no plasma.

\section{Barreiras e distribuição de compartimento de fluidos}

O volume e a composição de cada compartimento de fluido dependem das barreiras que o separam dos compartimentos vizinhos. A membrana celular separa os compartimentos intra e extracelulares e é impermeável a grandes moléculas hidrofílicas e partículas com cargas. Além da difusão passiva de certas moléculas, solutos podem atravessar as membranas celulares de diversas formas: Transporte ativo primário, transporte ativo secundário, canais de solutos, endocitose e exocitose.

A função de barreira do endotélio vascular é particularmente relevante no período perioperatório devido ao seu papel fundamental na manutenção do volume do fluido intravascular. O trauma tecidual cirúrgico tipicamente leva à perda do volume intravascular através da perda de sangue cirúrgico ou mudanças para outros compartimentos teciduais relacionadas a inflamação. O efeito fisiológico de fluido IV administrado para superar estas perdas e manter a oxigenação tecidual adequada é altamente dependente da patência de fluidos ao nível capilar.

A estrutura dos capilares varia dependendo da função do órgão subjacente. 0 tipo mais comum é o capilar não fenestrado, compreendendo a membrana basal 
Comparison between saline and buffered solutions: bibliographic review

Costa DAA; Silva WA

contínua e uma única camada de células endoteliais unidas por junções que são pontuadas por pausas. Estas fissuras intercelulares são o principal canal para o fluxo transcapilar de fluido. $O$ aspecto intravascular das células endoteliais está coberto por uma rede contínua de cadeias de glicosaminoglicanos associados a proteoglicanos ligados à membrana; e glicoproteínas, em conjunto formando a camada de glicocálice endotelial. Essa cobre fenestrações e fissuras intercelulares e tem uma espessura de até $1 \mu \mathrm{m}$. Além das suas funções na prevenção da adesão dos leucócitos e de plaquetas, ela emergiu como uma importante camada semipermeável contribuindo para a função de barreira endotelial. Água e eletrólitos podem se mover livremente através da camada subglicocálice (SGC). O volume da SGC pode ser tão alto quanto 700 a $1.000 \mathrm{~mL}$; por conseguinte, esse volume faz parte do volume intravascular e tem uma composição de eletrólitos em equilíbrio com o plasma, mas uma concentração muito mais baixa de proteínas devido à exclusão eficaz de moléculas maiores pelo glicocálice.

\section{Controle fisiológico do balanço hídrico geral}

Os rins têm vários papéis na manutenção da homeostase do corpo humano. Além de manter o estado acidobásico normal, os rins precisam regular a água corporal total e os solutos porque a ingesta diária de cada um é variável. A regulação imprópria pode resultar em pouca água corporal (desidratação celular) ou muita água (edema tissular). Isso também é válido quando administramos fluidos por via intravascular. Os líquidos intravenosos devem ser considerados como qualquer outro fármaco, sendo respeitada sua posologia, farmacocinética e farmacodinâmica. Por exemplo, pacientes internados na unidade de terapia intensiva ou que se apresentam no centro cirúrgico podem ter volume de LEC baixo, desidratação celular ou ambos como resultado de trauma importante, hemorragia, jejum ou desnutrição prolongados, bem como vômitos ou diarreia prolongados. A escolha da composição dos líquidos e sua velocidade de infusão são ditadas de acordo com o contexto clínico. O anestesiologista deve levar em consideração tais contextos clínicos e fisiopatológicos para selecionar o melhor fluido para realizar manutenção hídrica ou reposição quando houver déficit.

A concentração e o volume do LEC são mantidos pela sede e pelas ações hormonais do sistema renina-angiotensina-aldosterona (SRAA) e do hormônio antidiurético $(A D H)$ sobre os rins, que alteram a quantidade de água e de sódio excretados pela urina. O ADH é liberado (liberação não tônica) a partir da hipófise posterior em resposta a pequenas alterações (2-3\%) na tonicidade sérica ou $>10 \%$ de redução no volume circulante efetivo. O SRAA é ativado por hipotensão, pelo sistema nervoso simpático e pela redução no fornecimento de $\mathrm{Na}+$ para os rins. O resultado final é o aumento da sede (aumento da ingestão de água), aumento da retenção urinária de água e de sódio e vasoconstrição, que agem para manter o volume circulante efetivo e a perfusão dos órgãos vitais. 
Comparison between saline and buffered solutions: bibliographic review

Costa DAA; Silva WA

\section{FARMACOLOGIA DOS CRISTALOIDES}

Os cristaloides são soluções de eletrólitos na água. Eles podem ser classificados quanto à tonicidade (hipotônicos, isotônicos e hipertônicos) e presença de tampões (balanceados e não balanceados). Os cristaloides são indicados para substituição da água e eletrólitos livres, mas também podem ser utilizados para expansão de volumes. Referências antigas indicavam que apenas $20 \%$ dos cristaloides infundidos permaneciam no espaço intravascular, mas isso é extremamente questionado por grandes ensaios clínicos e pelo conhecimento atual da manipulação microvascular de fluidos, que sugerem que cristaloides isotônicos podem ter um efeito de expansão do volume intravascular maior do que isso, especialmente em pacientes com baixa pressão hidrostática capilar. Talvez até $70 \%$ de uma infusão de cristaloide permaneça no compartimento intravascular, no final de uma infusão contínua de 20 minutos, diminuindo para $50 \%$ após 30 minutos $^{11}$. Edema tecidual pode aumentar em tecidos complacentes, tais como o pulmão, intestino, e os tecidos moles, especialmente quando as soluções cristaloides são infundidas em paciente normovolêmicos. Infusões de grandes volumes de cristaloide estão associadas com um estado de hipocoagulabilidade causado por diluição de fatores anticoagulantes circulantes.

\section{Soluções Salinas}

Um dos cristaloides mais comumente usados na prática clínica é o $\mathrm{NaCl}$ a $0,9 \%$. Ele não foi inicialmente criado para uso in vivo, ainda assim entrou em uso clínico generalizado apesar de ter concentrações de sódio e cloro muito superiores às do plasma. A sua osmolaridade, calculada como a soma dos solutos presentes, é ligeiramente maior que a do plasma, embora a osmolalidade seja de $285 \mathrm{mOsm} / \mathrm{kg}$, muito próxima à do plasma. Ambos os íons permanecem no LEC após a infusão e pode ser considerado isotônico. Uma infusão de $2 \mathrm{~L}$ de $\mathrm{NaCl}$ a $0,9 \%$ conduz a um aumento do volume do LEC, diminuição dilucional do hematócrito e albumina, aumento das concentrações de cloro e potássio e diminuição de bicarbonato no plasma. A expansão do LEC é mais persistente do que com soluções cristaloides balanceadas. A diurese é mais tardia e menos extensa com salina isotônica e o excesso de carga de sal e água pode levar vários dias para ser excretado até mesmo para um sujeito saudável.

Existem, ainda, soluções hipertônicas de 1,8\%, 3\% e 7,5\% de $\mathrm{NaCl}$. Seus usos são restritos a determinadas situações clínicas e reposições pontuais de fluidos. Em concentrações superiores a 7,5\% podem causar lesão endotelial. Solução de $\mathrm{NaCl}$ a $11,7 \%$ pode ser utilizado como agente esclerosante.

\section{Soluções cristaloides balanceadas}

As soluções cristaloides balanceadas atualmente disponíveis possuem osmolaridade total inferior à osmolaridade do $\mathrm{NaCl}$ a $0,9 \%$, com menor concentração de 
Comparison between saline and buffered solutions: bibliographic review Costa DAA; Silva WA

$\mathrm{Na}^{+}$e concentração de $\mathrm{Cl}^{-}$muito mais baixas (ver tabela 1). A redução do teor aniônico é compensada pela adição de tampões aniônicos orgânicos estáveis, tais como lactato, gluconato ou acetato. A osmolalidade medida de soluções equilibradas ( $265 \mathrm{mOsm} / \mathrm{kg}$ ) é ligeiramente menor do que a do plasma, e elas são, portanto, ligeiramente hipotônicas. O lactato sofre oxidação predominantemente hepática ou gliconeogênese para produzir bicarbonato a uma taxa máxima de $200 \mathrm{mmol} / \mathrm{h}$. O acetato está normalmente presente em quantidades vestigiais no plasma sendo rapidamente oxidado pelo fígado, músculo e coração para produzir bicarbonato a um volume máximo de $300 \mathrm{mmol} / \mathrm{h}$. O metabolismo do gluconato está menos bem caracterizado, em termos de localização e cinética, mas ele é convertido em glicose como produto final.

A excreção de água e eletrólitos é mais rápida com soluções equilibradas do que com soluções salinas isotônicas. Isto se deve ao fato da redução transitória da tonicidade do plasma, o que suprime a secreção de $A D H$ e permite a diurese em resposta ao aumento do volume circulante intravascular. Cristaloides balanceados não reduzem a DIF plasmática no mesmo grau que soluções de $\mathrm{NaCl}$ e, por conseguinte, não provocam acidose.

Alguns efeitos negativos potenciais foram identificados com soluções cristaloides equilibradas. Soluções de Ringer Lactato contêm lactato racêmico (D- e L-), embora o Dlactato seja encontrado somente em quantidades vestigiais in vivo. A preocupação de que grandes doses de D-lactato possam estar associadas com encefalopatia e toxicidade cardíaca em pacientes com insuficiência renal não foi confirmada em estudos humanos em níveis plasmáticos obtidos com a utilização de solução de Ringer lactato racêmico. 0 metabolismo do D-lactato parece ser quase tão rápido quanto o do L-lactato. Preocupações sobre os efeitos do excesso de acetato exógeno foram levantadas pela síndrome bem reconhecida de intolerância ao acetato experimentada por pacientes em hemodiálise com dialisado à base de acetato. Os efeitos sistêmicos severos levaram a retirada do acetato de fluidos de diálise contemporâneos. O metabolismo do acetato em pacientes com disfunção renal em fase terminal e pela presença de substratos para oxidação - por exemplo, durante a acidose lática ou proteólise. Desta forma, é possível que pacientes criticamente doentes ou aqueles com doença renal avançada possam apresentar intolerância bioquímica ao acetato, embora esta possibilidade não tenha sido explorada em pacientes que recebem cristaloide equilibrado à base de acetato.

\section{ESTADO DOS LÍQUIDOS: AVALIAÇÃO E MONITORAÇÃO}

\section{Avaliação clínica convencional}

Hipovolemia, definida como volume sanguíneo circulante inadequado, resulta de depleção de volume (déficit de sódio no LEC) ou desidratação (déficit de água no LEC). A hipovolemia prolongada aumenta a morbimortalidade, assim como o excesso de administração de fluidos intravenosos também possui desfechos ruins. Os achados do 
Comparison between saline and buffered solutions: bibliographic review

Costa DAA; Silva WA

exame físico (distensão venosa jugular, turgor cutâneo, mucosas ressecadas, estertores respiratórios, B3 cardíaca, enchimento capilar, sinais vitais e hipotensão postural) e as alterações de peso corporal são ferramenta potencialmente úteis na avaliação do estado de volume.

\section{Avaliação clínica intraoperatória}

As medidas estáticas do estado de volume que são usadas no período intraoperatório incluem a pressão venosa central, a pressão de oclusão da artéria pulmonar e o diâmetro da veia cava inferior. Essas medidas estáticas, que eram consideradas medidas acuradas do volume intravascular, mostraram, por meio de evidências, não serem nem medidas precisas do volume intravascular e nem preditores precisos de responsividade aos líquidos. Devido ao fato de essas medidas serem fortemente influenciadas pela ventilação mecânica e alterações cardíacas, elas caíram em desuso como medidas primárias de estado volumétrico.

As medidas dinâmicas do estado de volume, contudo, levam em consideração flutuações do sistema cardiopulmonar ao longo de um período do tempo e mostraram ser mais acuradas para prever a responsividade aos líquidos. Uma medida dinâmica cada vez mais popular que tem se mostrado útil é a variação de pressão de pulso (VPP), que ocorre com ciclos respiratórios. A VPP é facilmente obtida a partir da onda de pulso da pressão arterial, desde que o paciente esteja ventilado mecanicamente e em ritmo sinusal. A VPP é calculada como uma porcentagem, com valores $>12 \%$ acima de pelo menos três ciclos respiratórios preditivos de hipovolemia e responsividade aos fluidos:

$$
V P P=\left(P P_{\text {máx }}-P P_{\text {min }} / P P_{\text {méd }}\right) \times 100
$$

Tanto a VPP quanto a medida relacionada de variação de volume sistólico (VVS) mostraram ser altamente preditivas da responsividade à reposição de líquidos, uma observação importante porque até $50 \%$ dos pacientes hipovolêmicos podem não responder à reposição de fluidos. Como a VPP e a VVS podem identificar de forma acurada os pacientes que irão se beneficiar da reposição de volume, isso as torna ferramentas clínicas valiosas.

\section{Manejo prático da fluidoterapia perioperatória}

Em cada etapa na jornada perioperatória, o médico deve decidir quanto e que tipo de fluido intravenoso é necessário. Quantidades de fluidos intravenosos podem ser administradas de duas formas principais: estimando-se os requisitos com base no peso do paciente, o que inclui as variações das fases cirúrgicas; e a natureza das perdas para calcular a dose necessária, ou por mediação direta de variáveis fisiológicas de um indivíduo, administrando-se fluido em quantidade suficiente para atingir uma melhora nessas variáveis fisiológicas, chamada terapia guiada por metas. 
Comparison between saline and buffered solutions: bibliographic review Costa DAA; Silva WA

A terapia com quantidades fixas de fluidos possui várias limitações quando são estudadas pois as diferentes definições de terapia restritiva e liberal dificulta a uniformização das pesquisas e comparação dos trabalhos. A abordagem guiada por metas vem se mostrando superior à tradicional de fixar a quantidade de fluido baseado no peso e porte do procedimento. A terapia guiada por metas possui o diferencial de ser dinâmica e se ajustar às necessidades do paciente de acordo com seu status clínico, débito cardíaco, momento cirúrgico e fluidoresponsividade ${ }^{12}$.

\section{Seleção do Cristaloide na Prática Clínica}

\section{Pediatria}

A indicação clínica mais recente reforça o uso de solução hipo-osmolar 0,2 ou $0,45 \%$ salina usualmente com 5 ou $10 \%$ de dextrose para pacientes neonatos até 1 mês de vida devido à função renal proporcionalmente menor nessa faixa etária quando comparada aos adultos. A adição de dextrose é considerada necessária pela rápida depleção das reservas hepáticas (naturalmente escassas). O risco de hipoglicemia é particularmente proeminente em crianças prematuras. Teoricamente, soluções salinas iso-osmolares causariam hipernatremia nessa população. Por outro lado, o risco de hiponatremia com o uso de soluções iso-osmolares permanece, pois níveis elevados de hormônio antidiurético, em decorrência do estresse fisiológico, podem causar retenção preferencial de água livre ${ }^{13}$. Uma meta análise recente comparando fluidos iso e hipoosmolares para manutenção da hidratação em pacientes pediátricos hospitalizados, sendo a maioria em pós-operatório, concluiu que fluidos iso-osmolares são superiores na prevenção de hiponatremia ${ }^{14}$. Isso sugere que, embora exista um risco de hipernatremia iatrogênica, o risco de hiponatremia, a partir de líquidos hiposmolares também permanece significativo. Com a maturidade renal, o risco de hipervolemia iatrogênica diminui e ainda há melhor manutenção dos eletrólitos plasmáticos pela capacidade renal de excreção. Nessa condição, os cristalóides balanceados (Ringer Lactato e Plasma Lyte $^{\circledR}$ ) passam a ser a melhor escolha, em decorrência da redução do risco de causar hipercloremia e acidose metabólica ${ }^{13}$.

Crianças que são submetidas à neurocirurgia são propensas a riscos maiores de hiponatremia, hipertensão intracraniana e edema cerebral. Logo, soluções hipotônicas costumam ser evitadas pelo risco teórico de causar tais complicações. Essa conduta esta sendo questionada há alguns anos e trabalhos recentes vem demonstrando desfechos melhores e menos complicações com o uso de soluções balanceadas em comparação à salina $0,9 \%$. Lima e colaboradores realizaram um ensaio clínico randomizado cujo objetivo foi avaliar o impacto do tipo de cristaloide (salina 0,9\% versus soluções balanceadas) utilizado em neurocirurgias de ressecção tumoral em pediatria. Concluiram que a infusão de salina $0,9 \%$ causou variações maiores no cloro sérico antes e depois da cirurgia. Observou-se ainda que as soluções balanceadas apresentam perfil 
Comparison between saline and buffered solutions: bibliographic review Costa DAA; Silva WA

mais seguro de eletrólitos e ácido-base quando comparado à salina 0,9\%. Não houve variações da pressão intracranina e/ou da avaliação do edema cerebral pelo cirurgião ${ }^{19}$.

\section{Transplante Renal}

No tocante ao transplante renal, o manejo hemodinâmico intraoperatório está associado à função do enxerto e complicações metabólicas. A reposição volêmica tem sido debatida no que diz respeito ao tipo e ao volume da solução empregada durante o perioperatório. Por isso, a escolha do fluido intravenoso utilizado no perioperatório tem recebido uma maior atenção, principalmente pelas repercussões que esse agente pode causar ao paciente, em especial ao renal crônico, que já apresenta importantes distúrbios metabólicos prévios e sobrecarga volêmica. Somado a isso, dunrate o transplante renal, ocorre o processo de isquemia e reperfusão do enxerto.

Classicamente, a solução salina $0,9 \%$ tinha sido utilizada em todos os pacientes renais crônicos, especialmente quando em estágio terminal, devido à ausência de potássio na solução. Entretanto, a maior concentração de cloro na solução salina $0,9 \%$ é associada a um risco aumentado de acidose metabólica hiperclorêmica, que, por conseguinte, associa-se à hipercalemia - por deslocamento do potássio para o meio extracelular. Soluções eletrolíticas balanceadas possuem menor concentração de cloro nas suas composições, sendo, portanto, mais fisiológicas. Exemplos dessas soluções são o Ringer Lactato e o Plasma-Lyte ${ }^{\circledR}$. A concentração reduzida de cloro pode evitar a necessidade de diálise por hipercalemia no pós-operatório imediato ${ }^{20}$.

Num estudo prospectivo em 2012 com 760 doentes críticos, Yunos e colaboradores compararam os cristaloides convencionais com as soluções balanceadas e encontrou uma menor taxa de injúria renal aguda, além de menor necessidade de terapia de substituição renal, no grupo que recebeu um regime de infusão com cloreto reduzido ${ }^{7}$. Pacientes com doença renal em fase terminal submetidos ao transplante renal podem ser especialmente propensos aos efeitos da hipercloremia devido a dois mecanismos: em primeiro lugar, devido à função limitada desse órgão com uma capacidade reduzida para se adaptar à hipercloremia; em segundo lugar, decorrente da possível hipercloremia induzida pela infusão de solução salina normal durante a fase perioperatória ${ }^{21}$.

Portanto, as soluções balancedas, as quais apresentam uma composição mais próxima ao plasma humano, são consideradas seguras para uso durante o transplante renal, quando se avalia o perfil do equilíbrio eletrolítico e ácido-básico.

No entanto, existem poucas evidências que avaliem desfecho a longo prazo, principalmente em relação ao sucesso do enxerto. 
Comparison between saline and buffered solutions: bibliographic review Costa DAA; Silva WA

\section{Neurocirurgia}

A barreira hematoencefálica possui seletividade quanto ao tamanho e carga das particulas que podem atravessá-la. A pressão oncótica do plasma é influenciada principalmente pelas proteínas, na sua grande maioria, com alto peso molecular. Isso faz com que o impacto das oscilações na pressão oncótica do plasma influenciem pouco o encéfalo e a osmolaridade seja mais importante na sua dinâmica de fluidos. Dessa forma, a escolha de soluções iso ou hiperosmolares é mais indicada para pacientes em que o objetivo seja a manutenção ou redução da pressão intracraniana (PIC), acreditando-se que soluções hiposmolares podem aumentar a $\mathrm{PIC}^{13}$.

Isso passou a ser discutido após ser observado os malefícios causados pela hipercloremia - insuficiência renal aguda, acidose metabólica, aumento de mortalidade e morbidade - que pode ser causada por essas soluções. Um estudo clínico randomizado controlado, único centro, comparou o uso de cristaloides balanceados e solução salina 0,9\% em 36 pacientes com hemorragia subaracnóide, nos quais a solução balanceada reduziu a incidência de hipercloremia sem aumentar o risco de hiponatremia ou hiposmolaridade ${ }^{15}$.

Nos pacientes com trauma cranioencefálico (TCE), a indicação de soluções iso e hiperosmolares foi unanimidade durante algum tempo. À luz do conhecimento mais atual, essa indicação passou a ser questionada devido ao risco aumentado de hipercloremia e suas compliações associadas. Cooper e colaboradores selecionaram aleatoriamente 229 pacientes com hipotensão (pressão arterial sistólica $<100 \mathrm{mmHg}$ ) e TCE severo para receberem uma infusão intravenosa rápida de solução salina hipertônica a 7,5\% (250 mL) ou Ringer Lactato $(250 \mathrm{~mL})$ no atendimento pré-hospitalar. A sobrevivência à alta hospitalar e função neurológica em 6 meses, avaliada pela escala extendida de desfecho de Glasgow (GOSE - Glasgow Outcome Scale Extended), foram similares em ambos os grupos $^{16}$.

Em pacientes com tumores intracranianos, durante o período perioperatório, a terapia hiperosmolar é comumente usada no intuito de reduzir o edema cerebral e colaborar diretamente com o procedimento cirúrgico. Raghava e colaboradores selecionaram pacientes com tumor intracerebral que seriam submetidos à craniotomia e o relaxamento cerebral foi observado pelo cirurgião em uma escala de 1 a 4 , sendo 1 perfeitamente relaxado e 4 edemaciado. Estes, foram divididos em 2 grupos: de um lado os que utilizaram soluções salinas hipertônicas a $3 \%$ e do outro lado o Manitol a $20 \%$, e foi demonstrado efetividade semelhante em ambos os grupos ${ }^{17}$. Outro estudo randomizado controlado comparou $5 \mathrm{ml} / \mathrm{kg}$ de manitol a $20 \%$ e $5 \mathrm{ml} / \mathrm{kg}$ de solução salina a $3 \%$ em tumores supratentoriais, sendo a PIC monitorada durante o procedimento e 30 minutos após a infusão da solução, e observou-se que a redução da PIC foi maior quando utilizou-se solução salina ${ }^{18}$. Contudo, esse dado precisa ser avaliado cuidadosamente. Não há comparação nos desfechos clínicos a médio e longo prazo e nem no que diz 
Comparison between saline and buffered solutions: bibliographic review

Costa DAA; Silva WA

respeito ao impacto do uso dessas soluções, bem como suas possíveis repercussões, como a hipercloremia que pode ser ocasionada e os desfechos finais. Logo, caso seja com a intenção única de reduzir PIC, parece que a solução salina hipertônica se apresenta superior ao manitol e às soluções isosmolares.

\section{CONCLUSÃO}

O conhecimento médico sobre anatomia, fisiologia e farmacologia sofreu importantes modificações e transformações ao longo da última década. Isso, associado à descoberta dos desfechos negativos da hipercloremia, trouxe uma nova percepção sobre o uso dos fluidos e suas indicações.

A teoria ácido-base de Steawart, a descoberta da lâmina subglicocálice e a terapia guiada pelo $\mathrm{pH}$ nos apresentaram uma nova abordagem dos distúrbios e farmacocinética dos fluidos intravenosos. A solução salina $0,9 \%$, erroneamente intitulada solução fisiológica, apesar de ainda ser a solução mais prescrita no mundo, vem tendo seu uso reduzido e indicações cada vez mais restritas. Os trabalhos mais atuais vêm reforçando os malefícios da hipercloremia e os benefícios de soluções balanceadas, cujas composições se aproximam das do plasma humano.

\section{REFERENCES}

1. Awad S, Allison SP, Lobo DN. The history of $0,9 \%$ saline. Clinical Nutrion Journal 2008;27:179-88.

2. Reddy S, Weinberg L, Young P. Crystalloid fluid therapy. Critical Care 2016; 20:59.

3. O'dell E, Tibby SM, Durward A et al. Hypercloremic is the dominant cause of metabolic acidosis in the postressucitaion phase of pediatric meningococcal sepsis. Crit Care Med 2007; 35:2390-4.

4. Noritomi DT, Soriano FG, Kellum JA. Metabolic acidosis in patients with severe sepsis and septic shock: a longitudinal quantitative study. Crit Care Med 2009; 37: 2733-9.

5. Nava E, Palmer RMJ, Moncada S. Inhibition of nitric oxide synthesis in septic shock: how much is benficial? Lancet 1991; 338: 8782-3.

6. Pedoto A, Nandi J, Oler A et al. Role of nitric oxide in acidosis-induced intestinal injury in anesthetized rats. Journal of Lab Clin Med 2001;138:270-6.

7. Pedoto A, Caruso JE, Nandi J et al. Acidosis stimulates nitric oxide production and lung damage in rats. Am K Respir Crit Care Med 1999; 159:397-402.

8. Filis C, Valiseiadis I, Koutsouku A. Hyperchloraemia in sepsis. Ann. Intensive Care 2018;8:43.

9. Miller RD. Miller Anestesia Tradução da 8a edição. Rio de Janeiro 2019.

10. Barash PG. Fundamentos de Anestesiologia Clínica. Porto Alegre 2017. 
Comparison between saline and buffered solutions: bibliographic review

Costa DAA; Silva WA

11. Hahn RG. Volume kinetics for infusion fluids. Anesthesiology 2010; 113:470-81.

12. Raghunathan k, Singh M, Lobo DN. Fluid Management in Abdominal Surgery. What, When, and When Not Administer. Anesthesiology Clin 2015; 33:51-64.

13. Padua AP, Macaraya JR, Dans LF. Isotonic versus hypotonic saline solution for maintenance intravenous fluid therapy in children: a systemic review. Pediatri Nephol 2015; 30:1163-72.

14. Wang J, Xu E, Xiao Y. Isotonic versus hypotonic maintenance IV fluids in hospitalized children: a meta-analysis. Pediatrics 2014;133:105-113.

15. Lehmann L, Bendel S, Uehlinger DE et al. Randomized, double-blind trial of the effect of fluid composition on electrolyte, acid-base, and fluid homeostasis in patient early after subarachnoid hemorrhage. Neurocrit Care 2013;18:5-12.

16. Cooper DJ, Myles PS, McDermott FT et al Prehospital hypertonic saline resuscitation of patients with hypotension and severe traumatic brain injury: a randomized controlled trial. JAMA 2004;291:1350-57.

17. Raghava A, Bidkar PU, Praksha MVSS et al. Comparasion of equiosmolar concentrarions of hypertonic saline and mannitol for intraoperative lax brain in patients undergoing craniotomy. Surg Neurol Int 2015; 6:73.

18. Ali A, Tetik A, Sabanci PA et al. Comparison of $3 \%$ hypertonic saline and $20 \%$ mannitol for reducing intracranial pressure in patients undergoing supratentorial brain tumor surgery: a randomized, double-blind clinical trial. J Neurosurg Anesthesiol 2018;30: 171-8.

19. Lima MF, Neville IS, Cavalheiro S. Balanced Crystalloid Versus Saline for Perioperative Intravenous Fluid Administration in Children Undergoing Neurosurgery: A randomized Clinical Trial. J Neurosurg Anesthesol 2018;31:30-35.

20. Wan S, Roberts MA, Mount P. Normal saline versus lower-chloride solutions for kidney transplantation. Cochrane Database of Syst Rev 2016;9:8.

21. Pfortmueller C, Funk GC, Potura E et al. An Acetate-Buffered Balanced Crystalloid Versus 0.9\% Saline in Patients with End-Stage Renal Disease Undergoing Cadaveric Renal Transplantation: A Prospective Randomized Controlled Trial. Wien Klin Wochenschr 2017; 129: 598-604. 